\title{
APPENDIX \\ Case Study to Healthcare Setting Matrix
}

\begin{tabular}{|c|c|c|c|}
\hline \# & CASE STUDY NAME & SETTING & TYPE \\
\hline 1 & A Summer Internship Journal & $\begin{array}{l}\text { Inpatient; Academic Medical } \\
\text { Center }\end{array}$ & Process Improvement \\
\hline 2 & Claims Payment Processing & Managed Care Organization & Process Improvement \\
\hline 3 & $\begin{array}{l}\text { Return-to-Work at a Home } \\
\text { Healthcare Agency }\end{array}$ & Home Health & Process Improvement \\
\hline 4 & $\begin{array}{l}\text { The Ophthalmologist Who } \\
\text { Could Not See (The Waste) }\end{array}$ & Ambulatory Surgery Center & Process Improvement \\
\hline 5 & Building a New IR Suite & $\begin{array}{l}\text { Inpatient; Interventional } \\
\text { Neuroradiology Department }\end{array}$ & Process Improvement \\
\hline 6 & $\begin{array}{l}\text { Emergency Department } \\
\text { Heroes }\end{array}$ & $\begin{array}{l}\text { Inpatient; Emergency } \\
\text { Department }\end{array}$ & Process Improvement \\
\hline 7 & $\begin{array}{l}\text { Hurricane Mia Hits the Patient } \\
\text { Access Call Center }\end{array}$ & Outpatient; Call Center & Patient Experience \\
\hline 8 & $\begin{array}{l}\text { The Cowboy Doctor's Patient } \\
\text { Experience }\end{array}$ & Inpatient; Rural Hospital & Patient Experience \\
\hline 9 & $\begin{array}{l}\text { Patient Navigation at the } \\
\text { Orthopedic Clinic }\end{array}$ & $\begin{array}{l}\text { Outpatient; Orthopedic and } \\
\text { Spine Clinic }\end{array}$ & Patient Experience \\
\hline 10 & $\begin{array}{l}\text { HCAHPS and the Quiet-at- } \\
\text { Night Measure }\end{array}$ & Inpatient & Patient Experience \\
\hline 11 & $\begin{array}{l}\text { Discharge Phone Calls } \\
\text { (En Español) }\end{array}$ & Inpatient & Patient Experience \\
\hline
\end{tabular}

(continued) 


\begin{tabular}{|c|c|c|c|}
\hline \# & CASE STUDY NAME & SETTING & TYPE \\
\hline 12 & $\begin{array}{l}\text { Patient Experience in Home } \\
\text { Care }\end{array}$ & Home Health & Patient Experience \\
\hline 13 & $\begin{array}{l}\text { Reducing Patient Falls: } \\
\text { The Sleuth Resident }\end{array}$ & $\begin{array}{l}\text { Inpatient; Academic Medical } \\
\text { Center }\end{array}$ & Patient Safety \\
\hline 14 & Sustaining Hand Hygiene & Ambulatory Surgery Centers & Patient Safety \\
\hline 15 & $\begin{array}{l}\text { A Warning Letter From the } \\
\text { State Regulator }\end{array}$ & Skilled Nursing Facility & Patient Safety \\
\hline 16 & Failure-to-Rescue & $\begin{array}{l}\text { Inpatient; Small Community } \\
\text { Hospital }\end{array}$ & Patient Safety \\
\hline 17 & CLIF's Medication Errors & Inpatient; Pharmacy & Patient Safety \\
\hline 18 & A Mom's Story of Sepsis & $\begin{array}{l}\text { Inpatient; Emergency } \\
\text { Department }\end{array}$ & Patient Safety \\
\hline 19 & Operating Room Recovery & Inpatient; Surgical Department & Performance Improvement \\
\hline 20 & $\begin{array}{l}\text { Opioid Overdoses in the } \\
\text { Emergency Department }\end{array}$ & $\begin{array}{l}\text { Inpatient; Emergency } \\
\text { Department }\end{array}$ & Performance Improvement \\
\hline 21 & $\begin{array}{l}\text { The Lunchroom: Physician } \\
\text { Engagement at a PCMH }\end{array}$ & Outpatient; Physician Practice & Performance Improvement \\
\hline 22 & The Fulfillment Affair & Managed Care Organization & Performance Improvement \\
\hline 23 & Composite Quality Score & $\begin{array}{l}\text { Inpatient; Multihospital Health } \\
\text { System }\end{array}$ & Performance Improvement \\
\hline 24 & $\begin{array}{l}\text { Both/And Thinking in } \\
\text { Readmission Prevention }\end{array}$ & $\begin{array}{l}\text { Inpatient; Case Management } \\
\text { Departments }\end{array}$ & Performance Improvement \\
\hline 25 & $\begin{array}{l}\text { Community Collaboration for } \\
\text { Suicide Prevention }\end{array}$ & Inpatient; Community Health & Performance Improvement \\
\hline
\end{tabular}

HCAHPS, Hospital Consumer Assessment of Healthcare Providers and Systems; PCMH, patient centered medical home. 